ARTICLE

Received 20 Jun 2012 | Accepted 22 Nov 2012 | Published 29 Jan $2013 \quad$ DOl: 10.1038/ncomms2323

\title{
Quantitative experimental determination of site-specific magnetic structures by transmitted electrons
}

\author{
Z.Q. Wang ${ }^{1}$ X.Y. Zhong ${ }^{1}$, R. Yu ${ }^{1}$, Z.Y. Cheng $^{1} \&$ J. Zhu ${ }^{1}$
}

Understanding the magnetic structure of materials on a nanometre scale provides fundamental information in the development of novel applications. Here we show a site-specific electron energy-loss magnetic chiral dichroism method, first experimentally demonstrating that the use of transmitted electrons allows us to quantitatively determine atomic sitespecific magnetic structure information on a nanometre scale. From one $\mathrm{NiFe}_{2} \mathrm{O}_{4}$ nanograin in composite films, we extract its atomic site-specific magnetic circular dichroism spectra and achieve the quantitative magnetic structure information, such as site-specific total magnetic moments and orbital to spin magnetic moment ratios, by constructively selecting the specific dynamical diffraction conditions in electron energy-loss magnetic chiral dichroism experiments. The site-specific electron energy-loss magnetic chiral dichroism method shows its unique ability for solving the site-specific magnetic structure at nanoscale resolution, compared with X-ray magnetic circular dichroism and neutron diffraction. This work opens a door to meet the challenge of exploring the magnetic structures of magnetic materials at the nanoscale using transmitted electrons.

\footnotetext{
${ }^{1}$ Beijing National Center for Electron Microscopy, Laboratory of Advanced Materials and Department of Materials Science and Engineering, Tsinghua University, Beijing 100084, China. Correspondence and requests for materials should be addressed to X.Z. (email: xyzhong@mail.tsinghua.edu.cn) or to J.Z. (email: jzhu@mail.tsinghua.edu.cn).
} 
W ith rapid progress in the fields of nanomagnetism, multiferroics and spintronics, a method to characterize local magnetic structure information of the nanostructures is of essential importance for understanding and designing the magnetic materials on a nanometre scale. However, the site-specific magnetic structure determination from a nanoscale region in complex materials is challenging.

Electron energy-loss magnetic chiral dichroism (EMCD) ${ }^{1}$ technique was invented as a new route for detecting the magnetic signals using the transmitted electron beam with a reported high spatial resolution ${ }^{2}$. The capability of EMCD in quantitatively solving magnetic structures is fundamentally dependent on the strong dynamical diffraction effects of fast electrons ${ }^{3,4}$. The dynamical diffraction effects of fast electrons had been constructively applied using the technique of atomlocation channelling enhanced micro-analysis ${ }^{5-8}$, especially the energy loss by channelled electrons technique ${ }^{9}$, to obtain the site-specific information, such as element contents, valence states and electronic configurations. Some theoretical EMCD calculations $^{10,11}$ and experimental work ${ }^{12}$ discussed about the site specificity of the EMCD signals. In this work, we experimentally demonstrate how we develop the site-specific EMCD method by adjusting dynamical diffraction conditions to modulate the magnetic circular dichroism (MCD) signals at non-equivalent crystallographic sites, and further using dynamical diffraction calculations to extract the site-specific MCD spectra with quantitative site-specific magnetic structure information obtained in an example of $\mathrm{NiFe}_{2} \mathrm{O}_{4}$ (NFO). Compared with previous EMCD works on the detection and analysis of ferromagnetic signals ${ }^{13,14}$, in this work we develop the sitespecific EMCD method as a unique technique, which is capable of quantitatively solving the unknown magnetic structures of the materials with non-equivalent crystallographic sites in a nanoscale level with a high site specificity. It is the first work to experimentally demonstrate that the transmitted electron source can be used to quantitatively determine magnetic structure in complex materials.

\section{Results}

Sample information. Here, we studied the NFO single crystal in a nanometre-scale composite film ${ }^{15,16}$. The low-magnification transmission electron microscope (TEM) image of the composite film and the selected area electron diffraction patterns of an NFO nanopillar in [110] zone axis are shown in Fig. 1. The NFO crystal has an inverse spinel structure and ferrimagnetic structure, which is written as $\left(\mathrm{Fe}^{3+} \downarrow\right)_{\mathrm{A}}\left(\mathrm{Ni}^{2+} \uparrow \mathrm{Fe}^{3+} \uparrow\right)_{\mathrm{B}} \mathrm{O}_{4}$ at its nominal stoichiometric ratio ${ }^{6,15}$. The ' $\mathrm{A}$ ' and ' $\mathrm{B}$ ' notations represent tetrahedral sites (tet) with downward-direction $(\downarrow)$ magnetic moments and octahedral sites (oct) with upward-direction ( $\uparrow$ ) magnetic moments, respectively. Owing to the magnetic field of $\sim 2 \mathrm{~T}$ of the objective lens exerted on the specimen, the upward direction $(\uparrow)$ refers to the direction parallel to the magnetic field, while the downward direction $(\downarrow)$ refers to the direction antiparallel to the magnetic field. Other necessary information about the sample is given in the section of Methods.

Dynamical diffraction effects on EMCD signals. The dynamical diffraction effects of fast electrons are mainly influenced by the incident and outgoing electron orientations, which change the distributions of incident and reciprocal waves, respectively ${ }^{17,18}$, and sample thickness is another key factor ${ }^{11,19}$. The dynamical diffraction conditions for fast electrons have essential impacts on the EMCD spectra, that is, to modulate the weights of MCD signals from different atoms at different crystallographic sites in the sample.
The influences of different incident electron orientations on EMCD signals are compared in Fig. 2a. Twenty-nine nanometerthick NFO crystal is oriented to the three-beam case with (004) systematic reflections excited (cf. Fig. 2a) and with $(2 \overline{2} 0)$ systematic reflections excited (cf. Fig. 2b). The electron energyloss spectroscopy (EELS) spectra were acquired at + and positions for EMCD measurements ${ }^{1}$. In Fig. 2 a, extremely strong EMCD signals (about 33\% difference for $\mathrm{Fe}$ and $42 \%$ for $\mathrm{Ni}$ ) were observed $^{13-14,20}$, which are critical for further quantitative analysis of magnetic structures. Here, we define that an EMCD signal of $\mathrm{Fe}$ or $\mathrm{Ni}$ has a 'L3 - L2 +' sign in case that it is negative on $L_{3}$ edge and positive on $L_{2}$ edge, while the contrary situation corresponds to a ' $\mathrm{L} 3+\mathrm{L} 2-$ ' sign. Comparing the EMCD signals in Fig. 2, the ' $\mathrm{L} 3-\mathrm{L} 2+$ ' sign for $\mathrm{Fe}$ in Fig. $2 \mathrm{a}$ is opposite to the ' $\mathrm{L} 3+\mathrm{L} 2-$ ' sign for Fe in Fig. $2 \mathrm{~b}$, while the 'L3-L2+' sign for $\mathrm{Ni}$ in Fig. $2 \mathrm{~b}$ is the same as that of $\mathrm{Ni}$ in Fig. 2a. The data points of the original EELS spectra in Fig. 2 are shown in Supplementary Fig. S1.

The changes in signs for the EMCD signals can be qualitatively explained by dynamical diffraction effects. In the three-beam case with (004) systematic rows excited, alternating (008) octahedral and tetrahedral planes (with $4 \mathrm{~B}$ and $2 \mathrm{~A}$ atoms, respectively, on each plane in a unit cell) are parallel to incident electrons as shown in the inset of Fig. 2a. The 4B planes with deeper potential energy wells are channelled along by electrons, and the MCD signals from $4 \mathrm{~B}$ planes are enhanced. The Fe and Ni EMCD signals with ' $\mathrm{L} 3-\mathrm{L} 2+$ ' sign reflect the dominating excitation of $2 \mathrm{Fe}^{3+} \uparrow$ and $2 \mathrm{Ni}^{2+} \uparrow$ atoms on $4 \mathrm{~B}$ planes. In the three-beam case with $(2 \overline{2} 0)$ systematic rows excited, alternating $4 \mathrm{~A}+4 \mathrm{~B}$ and $4 \mathrm{~B}$ planes are parallel to incident electrons (cf. inset of Fig. 2b). The enhanced $4 \mathrm{~A}+4 \mathrm{~B}\left(4 \mathrm{Fe}^{3+} \downarrow+2 \mathrm{Fe}^{3+} \uparrow+2 \mathrm{Ni}^{2+} \uparrow\right)$ planes with the dominating contributions from $\mathrm{Fe}^{3+} \downarrow$ and $\mathrm{Ni}^{2+} \uparrow$ atoms lead to 'L3 + L2 - ' sign Fe EMCD signal and 'L3 - L2 +' sign Ni EMCD signal. It is concluded that the different relative weights of MCD signals from octahedral (B) sites and tetrahedral (A) sites at the two dynamical diffraction conditions lead to the opposite signs for Fe EMCD spectra in Fig. 2a,b.

Moreover, the weights of MCD signals from octahedral sites and tetrahedral sites can also be modulated by varying the outgoing electron orientations. For example, with (004) systematic reflections excited, the incident angle $\theta_{\text {in,004 }}$ (angle between incident electrons and (004) crystal planes) for a $42-\mathrm{nm}$ thick NFO is tilted to be $2 \theta_{\mathrm{B}}\left(\theta_{\mathrm{B}}\right.$ is the Bragg angle for NFO (004) crystal planes) with tetrahedral sites enhanced for incident waves as shown in Fig. 3a. The EMCD spectra at different outgoing angles are investigated by locating the + and - detectors in five pairs of positions as shown in Fig. 3b, corresponding to outgoing angles of $2 \theta_{\mathrm{B}}, \theta_{\mathrm{B}}, 0,-\theta_{\mathrm{B}},-2 \theta_{\mathrm{B}}$. For reciprocal waves, it tends to enhance different sites at different outgoing angles, and the signs of experimental EMCD spectra (cf. in Fig. 3c) are observed changing with the outgoing angles. There shows no EMCD signal at outgoing angle of $2 \theta_{\mathrm{B}}$. The data points of the original EELS spectra in Fig. 3 are shown in Supplementary Fig. S2. The EMCD signal distribution on the diffraction plane for the above dynamical diffraction conditions was simulated depending on the theoretical dynamical diffraction calculation methods (Supplementary Fig. S3, Supplementary Discussion and Supplementary Methods), which was consistent with the signs of the experimental EMCD signals at different outgoing angles. The EMCD signals also vary with sample thickness owing to dynamical diffraction effects ${ }^{11,20}$ (see Supplementary Fig. S4). The sample with uniform and optimized thickness is selected to obtain EMCD spectra with high signal-to-noise ratio. To accurately measure thickness in a thin area via low-loss EELS ${ }^{21}$, which is essential for the EMCD quantitative analysis, the mean free path $^{21}$ is calibrated in the thick NFO area, where the 


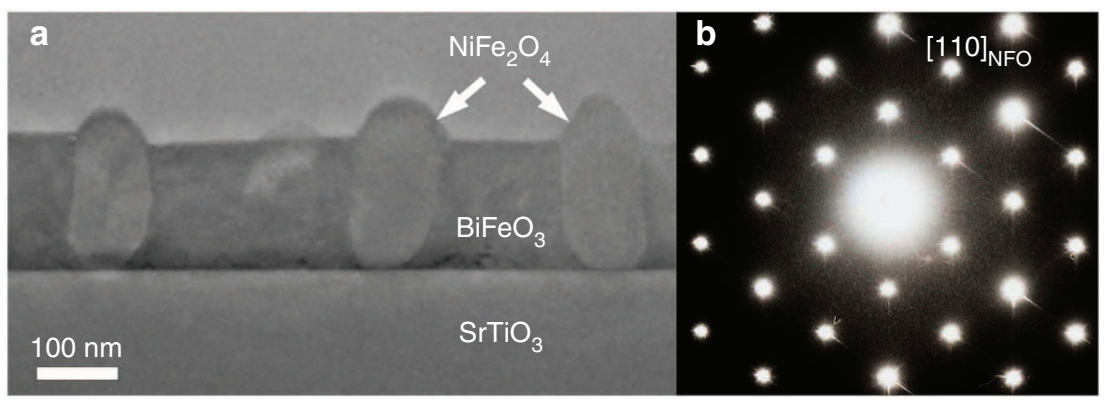

Figure 1 | Transmission electron microscopy (TEM) image and selected area electron diffraction (SAED) patterns of cross-sectional composite films. (a) Low-magnification TEM image for cross-section sample of hetero-epitaxial composite thin films formed by perovskite BFO and spinel NFO on the substrate of $\mathrm{SrTiO}_{3}$. (b) SAED patterns for one NFO crystal nanopillar tilted to the [110] zone axis.
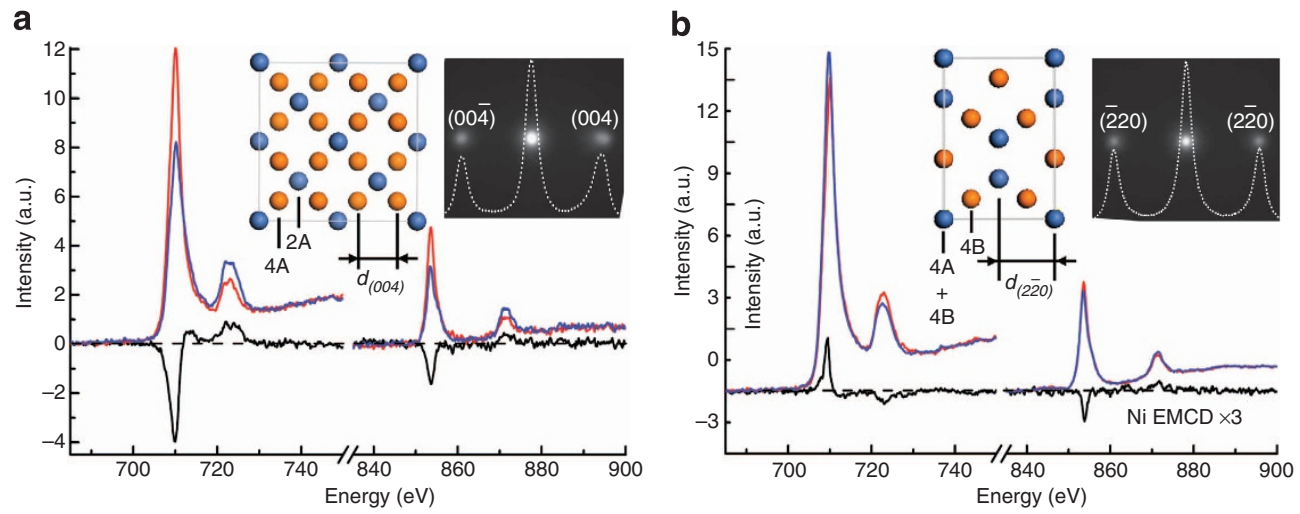

Figure 2 | Electron energy-loss magnetic chiral dichroism (EMCD) spectra measured in three-beam conditions with (004)/(2 20$)$ systematic reflections excited. (a) EMCD measurement on 29-nm-thick NFO sample in three-beam conditions with (004) systematic reflections excited where 2A (blue), 4B (orange), 2A, 4B ... crystal planes are parallel to the incident beam as shown in the inset of crystal lattice. The inset of diffraction plane shows the symmetrical excited diffraction spots and its intensity profile in experiment. The spectra are Fe and $\mathrm{Ni} L_{2,3}$ edges taken on two chiral positions ' + ' and ' - ' (shown in red and blue, respectively), and EMCD signal (the difference spectra, shown in black colour) for Fe and $N i L_{2,3}$ edges. (b) EMCD measurement on 29 -nm-thick NFO sample in three-beam conditions with $(2 \overline{2} 0)$ systematic rows excited where $4 A+4 B, 4 B, 4 A+4 B, 4 B \ldots$ crystal planes are parallel to incident beam. Fe $L_{2,3}$ edges and $\mathrm{Ni} L_{2,3}$ edges taken from ' + ' and ' - ' positions, and the corresponding EMCD spectra.
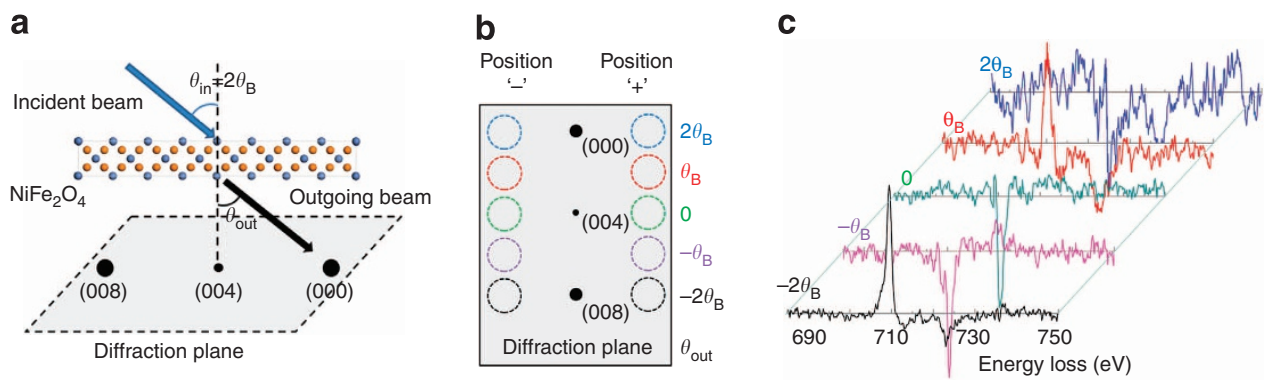

Figure 3 | Electron energy-loss magnetic chiral dichroism (EMCD) spectra of Fe with the incident angle $\theta_{\text {in, } 004}=2 \theta_{B}$ for $(004)$ systematic reflections at five different outgoing angles. A 42-nm-thick NFO sample was used. (a) The incident angle $\theta_{\mathrm{in}, 004}=2 \theta_{\mathrm{B}}$ for (004) systematic reflections was used. The sketch of incident beam, outgoing beam and the diffraction patterns. (b) The ' + ' and ' - ' entrance aperture was located at five pairs of positions corresponding to different outgoing angles of $2 \theta_{\mathrm{B}}, \theta_{\mathrm{B}}, 0,-\theta_{\mathrm{B},}-2 \theta_{\mathrm{B},}$ respectively. At a specific outgoing angle, ' + ' and '-' positions for EMCD measurement are chosen to be in mirror symmetry with respect to (004) reflection with a perpendicular distance of $0.8 g_{004}$. (c) Corresponding EMCD spectra of Fe taken on the five outgoing angles. The EMCD signal of oct Fe is dominating at outgoing angles of 0 and $-\theta_{B}$ leading to 'L3 - L2 + ' EMCD spectra, while the EMCD signal of tet Fe is dominating at outgoing angles of $\theta_{B}$ and $-2 \theta_{B}$ leading to ' $L 3+L 2-{ }^{\prime}$ EMCD spectra.

thickness is precisely determined using the convergent beam electron diffraction method. The high $\mathrm{S} / \mathrm{N}$ ratios in our EMCD experiments are attributed to the performance of the microscope, the high site specificity of EMCD signals for NFO and the optimized dynamical diffraction conditions (Supplementary Discussion).
Determination of magnetic structure information. In the following sections, we will demonstrate how to solve the magnetic structure in NFO by site-specific EMCD and testify its validity in determining magnetic structure information by comparing with the results by theoretical calculations and other experimental techniques. 


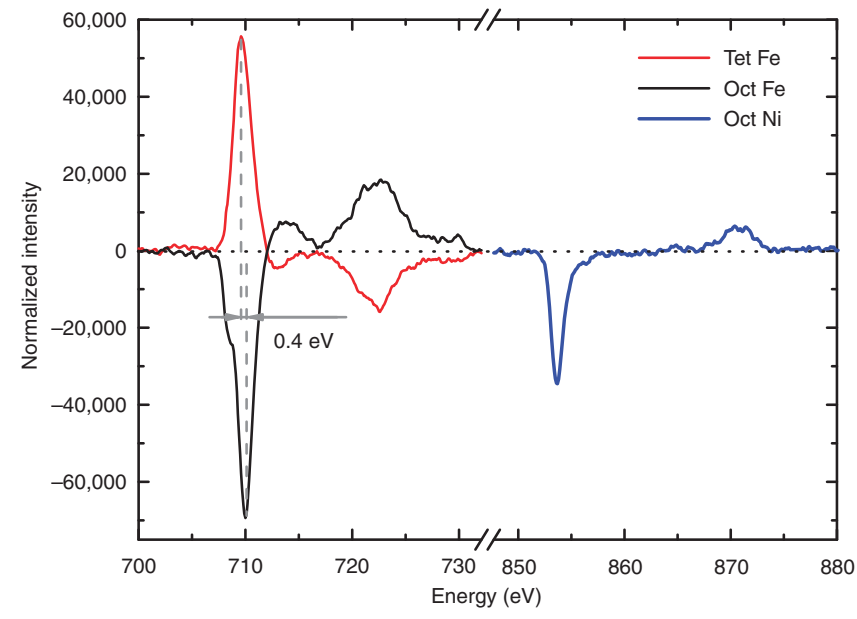

Figure 4 | Extracted site-specific magnetic circular dichroism pure spectra for oct Fe, oct $\mathbf{N i}$ and tet Fe. A possible energy shift of $0.4 \mathrm{eV}$ between oct Fe peak and tet Fe peak is a reflection of the chemical shift for different site symmetries.

To quantitatively distinguish the contributions of EMCD signals from atoms at oct and tet sites for further magnetic structure determination, dynamical diffraction calculations based on the theoretical framework ${ }^{10,19,22,23}$ were performed (Supplementary Methods). Generally, the EMCD spectrum at a certain dynamical diffraction condition can be written as:

$E M C D=a \cdot\left(\mu_{+}-\mu_{-}\right)_{\mathrm{oct}, \mathrm{Fe}}+b \cdot\left(\mu_{+}-\mu_{-}\right)_{\mathrm{tet}, \mathrm{Fe}}+a \cdot\left(\mu_{+}-\mu_{-}\right)_{\mathrm{oct}, \mathrm{Ni}}$

where $\mu_{+}$and $\mu_{-}$are similar to the X-ray absorption spectra for incident photons with left and right circular polarizations ${ }^{10}$, respectively (see the definitions of $\mu_{+}$and $\mu_{-}$in Supplementary Methods), and $\left(\mu_{+}-\mu_{-}\right)$represent the pure MCD spectra from atoms of oct $\mathrm{Fe}$, tet $\mathrm{Fe}$ and oct $\mathrm{Ni}$. The coefficients of $a$ for octahedral sites and $b$ for tetrahedral sites can be theoretically determined based on the crystallographic structure information of the material and the dynamical diffraction conditions (Supplementary Methods). The coefficients of $a$ and $b$ vary with dynamical diffraction conditions corresponding to different weights of MCD spectra from the atoms at different sites.

It is calculated that the ratio of $a$ (oct) to $b$ (tet) for NFO in Fig. 2a is $1:-0.44$ (Supplementary Fig. S5), indicating that the MCD spectra from both oct Fe and tet Fe positively contribute to the strong experimental 'L3 - L2 +' sign Fe EMCD signal. In Fig. $2 b$, the ratio of $a$ to $b$ is $0.25: 1$, (Supplementary Fig. S6) implying that the 'L3 $+\mathrm{L} 2$-' sign EMCD signal of Fe mainly arises from the dominating MCD signal from tet Fe. The strong EMCD spectra in Fig. 2 are attributed to the enhancement effects under these dynamical diffraction conditions.

Based on theoretically calculated coefficients of $a$ and $b$, the site-specific MCD spectra of $\left(\mu_{+}-\mu_{-}\right)_{\text {oct,Fe }},\left(\mu_{+}-\mu_{-}\right)_{\text {tet,Fe }}$ and $\left(\mu_{+}-\mu_{-}\right)_{\text {oct,Ni }}$ are extracted from a series of experimental EMCD spectra at different dynamical diffraction conditions via statistical method of least square fitting that achieves the minimal residual errors, as shown in Fig. 4 (Supplementary Fig. S7 and Supplementary Methods). As a comparison, the site-specific MCD spectra cannot be experimentally obtained from X-ray magnetic circular dichroism (XMCD) except by simulating the spectra via multiplet calculations ${ }^{24}$. Even if at the beginning we knew nothing about the magnetic structure of NFO, the sitespecific MCD spectra can give a clear demonstration of the ferrimagnetic structure of NFO, as the coefficients $a$ and $b$ are just calculated out using the knowledge of crystallographic structure information and dynamical diffraction condition. The general features of site-specific EMCD spectra are consistent with the previous simulated MCD spectra of oct Fe and tet Fe via multiplet calculations ${ }^{24,25}$. A possible energy shift of $0.4 \mathrm{eV}$ between EMCD peaks of oct Fe and tet Fe in Fig. 4 is a reflection of the chemical shift for different site symmetries, which agrees with the simulated value ${ }^{25}$.

Using the sum rules $4,11,26,27$, which allow a quantitative assessment of spin and orbital magnetic moments from the site-specific MCD spectra (Supplementary Methods), we further calculate the site-specific magnetic moments as shown in Table 1. Compared with the results obtained by XMCD, neutron diffraction and first-principle calculation ${ }^{20,28-30}$, site-specific EMCD method shows the unique capability of solving magnetic structures experimentally and simultaneously achieving very comprehensive magnetic information. The orbital to spin magnetic moment ratios $\left(m_{\mathrm{L}} / m_{\mathrm{S}}\right.$ ratios) for oct $\mathrm{Fe}$ and tet $\mathrm{Fe}$ are first reported, which are hardly acquired via other techniques. The $m_{\mathrm{L}} / m_{\mathrm{S}}$ ratio for oct $\mathrm{Ni}$ measured by EMCD is close to that measured by $\mathrm{XMCD}^{20}$. It is the first time to use EMCD method to determine site-specific total magnetic moments (the sum of spin and orbital magnetic moments). The obtained sitespecific total magnetic moment values are somewhat smaller but comparable with those measured by neutron diffraction and first-principle calculations. Besides, total magnetic moment in a unit cell of NFO by EMCD is close to that by macroscopic magnetic measurement ${ }^{31}$. The errors for the site-specific magnetic values are discussed in detail in Supplementary Table S1 and Supplementary Discussion.

\section{Discussion}

Compared with other magnetic-characterization techniques, such as XMCD and neutron diffraction ${ }^{30,32}$, site-specific EMCD method has its unique advantages. XMCD lacks the ability of distinguishing magnetic contributions from different atomic sites. Neutron diffraction is difficult to distinguish magnetic contributions from atoms of different elements with close atomic form factor randomly distributed in a same site, and its spatial resolution is rather limited. In comparison, as shown in this work, site-specific EMCD method can achieve site-specific and element-specific magnetic information, as well as distinguish the orbital and spin magnetic moments in a nanoscale level, which displays its unique values.

Generally, this work opens the door to quantitatively determine the site-specific magnetic structures for a wide range of magnetic materials in a nanoscale level using fast electrons. In the method of site-specific EMCD, dynamical diffraction effects of electrons are constructively used for tuning the site specificity through changing the Bloch wave fields, and considerably improve the EMCD signals. In the case of NFO, site-specific (oct Fe, tet Fe and oct $\mathrm{Ni}$ ) total magnetic moments and $m_{\mathrm{L}} / m_{\mathrm{S}}$ ratios were semiquantitatively and quantitatively, respectively, determined. The $m_{\mathrm{L}} / m_{\mathrm{S}}$ ratios for oct $\mathrm{Fe}$ and tet $\mathrm{Fe}$ are for the first time reported here. It is first demonstrated that the transmitted electron source is capable to quantitatively determining magnetic structure in complex materials. Given the ability of quantitatively determining magnetic structures with comprehensive magnetic information in a nanometre-scale region, site-specific EMCD may benefit much to the research and application of magnetic materials as a unique magnetic-characterization tool.

\section{Methods}

Sample preparation. The sample in our experiment is a cross-section sample of hetero-epitaxial composite thin film formed by perovskite $\mathrm{BiFeO}_{3}(\mathrm{BFO})$ and spinel $\mathrm{NFO}^{15,16}$. The low-magnification morphology image is shown in Fig. 1a. The composite thin film was grown by pulsed laser deposition at $700{ }^{\circ} \mathrm{C}$ on (001)$\mathrm{SrTiO}_{3}$ single-crystal substrate ${ }^{15}$. The ferrimagnetic NFO and antiferromagnetic BFO single-crystal nanopillars with the size of about $100 \mathrm{~nm}$ grow perpendicular to the substrate. Using high-resolution transmission electron microscopy and selected 
Table 1 | Magnetic information for $\mathrm{NiFe}_{2} \mathrm{O}_{4}$ via site-specific EMCD compared with that obtained from other techniques.

\begin{tabular}{|c|c|c|c|c|c|}
\hline & EMCD & XMCD & Calculation & Neutron diffraction & Macro-measure \\
\hline$m_{\mathrm{L}} / m_{\mathrm{S}}$ (oct Fe) & $0.01 \pm 0.02$ & & & & \\
\hline$m_{\mathrm{L}} / m_{\mathrm{S}}($ tet $\mathrm{Fe})$ & $0.06 \pm 0.02$ & & & & \\
\hline$m_{\mathrm{L}} / m_{\mathrm{S}}$ (oct $\left.\mathrm{Ni}\right)$ & $0.24 \pm 0.02$ & $\begin{array}{c}0.135 \pm 0.035 \text { (ref. 20) } \\
0.17 \pm 0.055 \text { (ref. 20) }\end{array}$ & & & \\
\hline$M_{\mathrm{oct}, \mathrm{Fe}}$ & $3.5 \pm 0.6$ & & 3.70 (ref. 28), 4.1 (ref. 29), 4.11* & 4.73 (ref. 32) & \\
\hline$M_{\mathrm{tet}, \mathrm{Fe}}$ & $-2.9 \pm 0.3$ & & -3.24 (ref. 28), -4.1 (ref. 29), $-4.00^{\star}$ & -4.86 (ref. 32) & \\
\hline$M_{\text {oct }, \mathrm{Ni}}$ & $1.7 \pm 0.2$ & & 1.38 (ref. 28), 2.2 (ref. 29), 1.58* & 2.22 (ref. 32) & \\
\hline$M_{\text {oct }, N V} / M_{\text {oct }, F e}$ & $0.49 \pm 0.04$ & & 0.37 (ref. 28), 0.54 (ref. 29), 0.38* & 0.46 (ref. 16) & \\
\hline$M_{\text {tot }}$ for $\mathrm{NiFe}_{2} \mathrm{O}_{4}$ & $2.3 \pm 0.9$ & & 1.74 (ref. 28), 2.2 (ref. 29), 2.0* & 2.1 (ref. 32) & 2.3 (ref. 30) \\
\hline
\end{tabular}

EMCD, energy-loss magnetic chiral dichroism; XMCD, X-ray magnetic circular dichroism. $m_{\llcorner} / m_{\mathrm{S}}$ refers to the orbital to spin magnetic moment ratio. $M_{\text {oct, }, \mathrm{Fe}}, M_{\text {tet, }, \mathrm{Fe}}$ and $M_{\mathrm{oct}, \mathrm{Ni}}$ are the total magnetic moments (the sum of spin and orbital magnetic moments) of oct Fe, tet Fe and oct $\mathrm{Ni}$ in the units of $\mu_{\mathrm{B}}$ per atom. $\mathrm{M}_{\text {tot }}$ for $\mathrm{NiFe}_{2} \mathrm{O}_{4}$ are the total magnetic moments for NiFe $\mathrm{O}_{4}$ in a unit cell. In the column of 'calculation', the total magnetic moments from ref. 29 were calculated from the spin magnetic moments and orbital magnetic moments mentioned in ref. 29 , and according to its context, almost all orbital magnetic moments are attributed to $\mathrm{Ni}^{2+}$. 'Calculation' means the 'first principle calculation', in which column the data with asterisks $\left({ }^{*}\right)$ are the results obtained by ourselves through density functional theory calculations. 'Macro-measure' refers to the macroscopic-measured saturation magnetic moments by the pondermotor method ${ }^{30}$

area electron diffraction, it is indicated that the NFO and BFO nanopillars not only grow epitaxial to the substrate in [001] orientation but also grow epitaxial to each other with the interface between them lying in the $\{110\}$ orientation. When the NFO nanopillar is tilted to the [110] zone axis, $(220)$ and (002) reflections perpendicular to each other are excited in the diffraction plane, as shown in Fig. $1 \mathrm{~b}$. In the EMCD measurements, $(2 \overline{2} 0)$ or (004) systematic reflections are, respectively, excited.

$\mathrm{BFO}$ has a rhombohedrally distorted perovskite structure with antiferromagnetic magnetic structure, and exhibit weak ferromagnetism owing to a canted spin structure $^{33}$. NFO has an inverse spinel structure. In spinel crystallography, NFO can be written as $\left(\mathrm{Fe}^{3+}\right)_{\mathrm{A}}\left(\mathrm{Ni}^{2+} \mathrm{Fe}^{3+}\right)_{\mathrm{B}} \mathrm{O}_{4}$ in its nominal stoichiometric ratio. $\mathrm{NFO}$ is nearly a complete inverse spinel in that most of the Ni atoms occupy the octahedral sites, with $\delta \approx 0.01$ in the formula $\left(\mathrm{Fe}_{1-\delta} \mathrm{Ni}_{\delta}\right)_{\mathrm{A}}\left(\mathrm{Fe}_{1+\delta} \mathrm{Ni}_{1-\delta}\right)_{\mathrm{B}} \mathrm{O}_{4}$ (ref. 34). Therefore, we do the calculation under the assumption that NFO is a complete inverse spinel with an error $<1 \%$. The energy dispersive spectroscopy measurements under non-channelling conditions show that the atomic ratio between $\mathrm{Ni}$ and $\mathrm{Fe}$ in our sample is 1:2.

Acquisition of EMCD spectra. The EELS and EMCD experiments were performed using a post-column Gatan Tridium system attached to the FEI Titan 80-300 TEM with Cs corrector, operating at $300 \mathrm{kV}$ with an energy resolution of about $0.7 \mathrm{eV}$. The objective lens exerted a magnetic field of $\sim 2 \mathrm{~T}$ on the specimen, whose magnetization is therefore saturated. In the EMCD measurements, nearly parallel beam (convergent angle $<0.5 \mathrm{mrad}$ ) with the probe size of around $50 \mathrm{~nm}$ in diameter was used to illuminate the region inside an individual NFO nanopillar with uniform thickness. The specimen was tilted to the desired dynamical diffraction conditions (Supplementary Fig. S8 and Supplementary Methods). The incident conditions were precisely determined as shown in Supplementary Materials. The outgoing conditions were determined by the relative positions of diffraction spots to the entrance aperture, and the collection angle is about $4 \mathrm{mrad}$. The error in aperture position at diffraction plane is about $0.05 \mathrm{mrad}$ and negligible owing to the strict geometrical construction. The data processing of the EELS spectra, including background subtraction from the pre-edge area, normalization by the post-edge area and Fourier-ratio deconvolution ${ }^{35}$ using a zero-loss modifier function in Digital Micrograph, is discussed in detail in Supplementary Fig. S9 and Supplementary Methods.

Calculations. To obtain the dynamical coefficients for EMCD, the bw software ${ }^{23}$ and the home-made codes were used based on the theoretical calculation frame ${ }^{10,19}$. The site-specific MCD spectra were extracted by least square fitting method from a series of EMCD spectra with the minimal residual errors for different dynamical diffraction conditions. Based on sum rules for MCD spectra, magnetic values are calculated for each atom in $\mathrm{NFO}^{26,27}$. Calculations of the magnetic structure of NFO were carried out using the projector augmented-wave method ${ }^{36}$ within the density functional theory, as implemented in the VASP code

${ }^{37}$. Detailed calculation descriptions and error analysis can be found in Supplementary Methods.

\section{References}

1. Schattschneider, P. et al. Detection of magnetic circular dichroism using a transmission electron microscope. Nature 441, 486-488 (2006).

2. Schattschneider, P. et al. Detection of magnetic circular dichroism on the two-nanometer scale. Phys. Rev. B 78, 104413-104417 (2008).

3. Warot-Fonrose, B. et al. Mapping inelastic intensities in diffraction patterns of magnetic samples using the energy spectrum imaging technique.

Ultramicroscopy 108, 393-398 (2008).
4. Lidbaum, H. et al. Quantitative magnetic information from reciprocal space maps in transmission electron microscopy. Phys. Rev. Lett. 102, 037201 (2009).

5. Taftø, J. \& Krivanek, O. L. Site-specific valence determination by electron energy-loss spectroscopy. Phys. Rev. Lett. 48, 560-563 (1982).

6. Tatsumi, K., Muto, S., Nishida, I. \& Rusz, J. Site-specific electronic configurations of Fe $3 d$ states by energy loss by channeled electrons. Appl. Phys. Lett. 96, 201911 (2010).

7. Tatsumi, K. \& Muto, S. Local electronic structure analysis by site-selective ELNES using electron channeling and first-principles calculations. J. Phys: Condens. Matter 21, 104213 (2009).

8. Taftø, J. \& Zhu, J. Electron energy loss near edge structure (ELNES), a potential technique in the studies of local atomic arrangements. Ultramicroscopy $\mathbf{9}$, 394-354 (1982).

9. Krivanek, O. L., Disco, M. M., Tafto, J. \& Spence, J. C. H. Electron energy loss spectroscopy as a probe of the local atomic environment. Ultramicroscopy $\mathbf{9}$, 249-254 (1982).

10. Calmels, L. \& Rusz, J. Momentum-resolved EELS and EMCD spectra from the atomic multiplet theory: application to magnetite. Ultramicroscopy 110, 1042-1045 (2010).

11. Calmels, L. \& Rusz, J. Atomic site sensitivity of the energy loss magnetic chiral dichroic spectra of complex oxides. J. Appl. Phys 109, 07D328 (2011).

12. Ennen, I. et al. Site-specific chirality in magnetic transitions. J. Magn. Magn. Mater. 324, 2723-2726 (2012).

13. Calmels, L. et al. Experimental application of sum rules for electron energy loss magnetic chiral dichroism. Phys. Rev. B 76, 060409 (2007).

14. Rubino, S. et al. Energy loss magnetic chiral dichroism (EMCD): magnetic chiral dichroism in the electron microscope. J. Mater. Res 23, 2582-2590 (2008).

15. Zhan, Q. et al. Structure and interface chemistry of perovskite-spinel nanocomposite thin films. Appl. Phys. Lett 89, 172902 (2006).

16. Zheng, $\mathrm{H}$. et al. Multiferroic $\mathrm{BaTiO}_{3}-\mathrm{CoFe}_{2} \mathrm{O}_{4}$ nanostructures. Science 303, 661-663 (2004).

17. Taftø, J. \& Krivanek, O. L. Characteristic energy losses from channeled $100 \mathrm{keV}$ electrons. Nucl. Instr. Meth. 194, 153-158 (1982).

18. Kainuma, Y. The theory of Kikuchi patterns. Acta Cryst. 8, 247-257 (1955).

19. Rusz, J., Rubino, S. \& Schattschneider, P. First-principles theory of chiral dichroism in electron microscopy applied to $3 d$ ferromagnets. Phys. Rev. B 75, 214425 (2007).

20. van der Laan, G. et al. Orbital polarization in $\mathrm{NiFe}_{2} \mathrm{O}_{4}$ measured by Ni- $2 p \mathrm{x}$-ray magnetic circular dichroism. Phys. Rev. B 59, 4314-4321 (1999).

21. Egerton, R. F. Electron Energy-Loss Spectroscopy in the Electron Microscope 2nd edn (Plenum, New York, 1996).

22. Löffler, S. \& Schattschneider, P. A software package for the simulation of energy-loss magnetic chiral dichroism. Ultramicroscopy 110, 831-835 (2010),

23. Schattschneider, P., Nelhiebel, M., Schenner, M., Grogger, W. \& Hofer, F. Diffraction effects in inner-shell ionization edges. J. Microsc. 183, 18-26 (1996).

24. Brice-Profeta, S. et al. Magnetic order in $\gamma-\mathrm{Fe}_{2} \mathrm{O}_{3}$ nanoparticles: a XMCD study. J. Magn. Magn. Mater. 288, 354-365 (2005).

25. Kuiper, P. et al. $\mathrm{Fe} \mathrm{L}_{2,3}$ linear and circular dichroism of $\mathrm{Fe}_{3} \mathrm{O}_{4}$. J. Electron Spectrosc 86, 107-113 (1997).

26. Carra, P., Thole, B. T., Altarelli., M. \& Wang, X. X-ray circular dichroism and local magnetic fields. Phys. Rev. Lett. 70, 694-697 (1993).

27. O’Brien, W. L. \& Tonner, B. P. Orbital and spin sum rules in x-ray magnetic circular dichroism. Phys. Rev. B 50, 672-681 (1994). 
28. Pénicaud, M., Siberchicot, B., Sommers, C. B. \& Kübler, J. Calculated electronic band structure and magnetic moments of ferrites. J. Magn. Magn. Mater. 103, 212-220 (1992).

29. Szotek, Z. et al. Electronic structures of normal and inverse spinel ferrites from first principles. Phys. Rev. B 74, 174431 (2006).

30. Rao, L. M. Neutron investigation of ferrites. Bull. Mater. Sci. 7, 303-320 (1985).

31. Gorter, E. W. Saturation magnetization and crystal chemistry of ferrimagnetic oxides. Philips Res. Rep. 9, 321 (1954).

32. Richter, M. C. et al. $\mathrm{NiFe}_{2} \mathrm{O}_{4}$ and $\mathrm{Fe}_{3} \mathrm{O}_{4}$ studied by XMCD and resonant photoemission. Eur. Phys. J. Special Topics 169, 175-180 (2009).

33. Wang, J. et al. Epitaxial $\mathrm{BiFeO}_{3}$ multiferroic thin film heterostructures. Science 299, 1719-1722 (2003).

34. Youssef, S. I., Natera, M. G., Begum, R. J., Srinivasan, B. S. \& Murthy, N. S. Polarised neutron diffraction study of nickel ferrite. J. Phys. Chem. Solids 30, 1941-1948 (1969).

35. Rusz, J. et al. Influence of plural scattering on the quantitative determination of spin and orbital moments in electron magnetic chiral dichroism measurements. Phys. Rev. B 83, 132402 (2011).

36. Blochl, P. E. Projector augmented-wave method. Phys. Rev. B 50, 17953-17979 (1994).

37. Kresse, G. \& Furthmüller, J. Efficient iterative schemes for $a b$ initio total-energy calculations using a plane-wave basis set. Phys. Rev. B 54, 11169-11186 (1996).

\section{Acknowledgements}

This work is financially supported by National 973 Project of China (2009CB623701) and Chinese National Nature Science Foundation (51001064, 51071092, 50831001 and 50971075). This work made use of the resources of the Beijing National Centre for Electron Microscopy. We are grateful to Profs Z.H. Zhang, J. Yuan, X.Q. Pan, D.S. Wang,
Dr. Lv N., Dr. L. Xie, Mr. Y. Xia, D.S. Song and Z.Y. Wang for helpful discussions, and Profs S.P. Crane, R. Ramash and Q. Zhan for providing the thin films and preparing the TEM specimen. We also thank Dr. S. Löffler for offering bw 0.1rcl code. Z.Q.W. also thanks the support of Gatan China Scholarship for this work.

\section{Author contributions}

J.Z. proposed and supervised this project, as well as contributed to the result analyses and manuscript writting. Z.Q.W. and X.Y.Z. suggested the method of adjusting dynamical diffraction conditions to enhance site-specific EMCD signals, conceived, performed the experiments, analysed the data and wrote the manuscript. Z.Q.W. developed the codes for parts of the theoretical calculations. R.Y. suggested to study the magnetism of the spinel-type nickel ferrite NFO, performed the density functional theory calculations, and participated in the experimental design and manuscript writting. Z.Y.C. helped substantially in performing the experiments. All authors discussed the results and commented on the manuscript.

\section{Additional information}

Supplementary Information accompanies this paper on http://www.nature.com/ naturecommunications

Competing financial interests: The authors declare no competing financial interests.

Reprints and permission information is available online at http://npg.nature.com/ reprintsandpermissions/

How to cite this article: Wang, Z.Q. et al. Quantitative experimental determination of site-specific magnetic structures by transmitted electrons. Nat. Commun. 4:1395 doi: 10.1038/ncomms2323 (2013). 